\title{
Cost Bounds \\ of Multicast Light-Trees in WDM Networks
}

\author{
Fen Zhou ${ }^{1}$, Miklós Molnár ${ }^{1}$, Bernard Cousin $^{2}$, and Chunming Qiao ${ }^{3}$ \\ 1 IRISA / INSA, Campus de Beaulieu, Rennes 35042, France \\ \{fen.zhou, molnar\}@irisa.fr \\ 2 IRISA / University of Rennes 1, Campus de Beaulieu, Rennes 35042, France \\ bernard.cousin@irisa.fr \\ 3 Computer Science Department, State University of New York at Buffalo, USA \\ qiao@computer.org
}

\begin{abstract}
The construction of light-trees is one principal subproblem for multicast routing in sparse splitting Wavelength Division Multiplexing (WDM) networks. Due to the light splitting constraint and the absence of wavelength converters, several light-trees may be required to establish a multicast session. However, the computation of optimal multicast light-trees is NP-hard. In this paper, we study the wavelength channel cost (i.e., total cost) of the light-trees built for a multicast session. An equal cost of 1 unit hop-count cost is assumed over all the fiber links in the network. We prove that the total cost of a multicast session is tightly lower limited to $K$ and upper bounded to (1) $K(N-K)$ when $K<\frac{N}{2}$; (2) $\frac{N^{2}-1}{4}$ or $\frac{N^{2}}{4}$ respectively when $K \geq \frac{N}{2}$ and $N$ is odd or even, where $K$ is the number of destinations in the multicast session and $N$ is the number of nodes in the network. Classical sparse splitting multicast routing algorithms such as Reroute-to-Source and Member-Only [3] also follow these bounds. And particularly in WDM rings, the optimal multicast light-tree has a cost inferior to $N-\left\lceil\frac{N}{K+1}\right\rceil$.
\end{abstract}

Keywords: WDM Networks, All-Optical Multicast Routing (AOMR), Light-trees, Sparse Splitting, Cost Bounds.

\section{Introduction}

Multicast routing in WDM networks is to determine a set of lightpaths on a physical topology from a source to a set of destinations involved in a multicast communication. The light-tree concept is introduced in [1] to minimize the number of wavelength channels and transceivers used for a multicast session. Branching nodes in a light-tree should be equipped with light splitters. However, in sparse splitting [2] WDM networks, there are two kinds of nodes: multicast capable nodes (MC [2], i.e. nodes equipped with light splitters) and multicast incapable nodes (MI 2], i.e. nodes without light splitters). An MC node is capable of replicating the data packets in the optical domain via light splitting and sends them to several ports, while an MI node cannot split but generally has 
the Tap-and-Continue ( $\mathrm{TaC}$ 4]) capacity which permits to tap a small amount of optical power from the incoming light beam and forward it to only one outgoing port. Although one tree is sufficient to span all the multicast destinations in a network without constraint, minimizing the cost of the multicast tree is already a Steiner-Problem which is proven to be NP-complete. In face of sparse splitting, lack of wavelength converters, as well as continuous wavelength and distinct wavelength constraints [5, one light-tree may not be enough to cover the entire multicast group members but several ones may be required, i.e., a light-forest [3. As a result, it is even harder to optimize the total number of wavelength channels used for a multicast session.

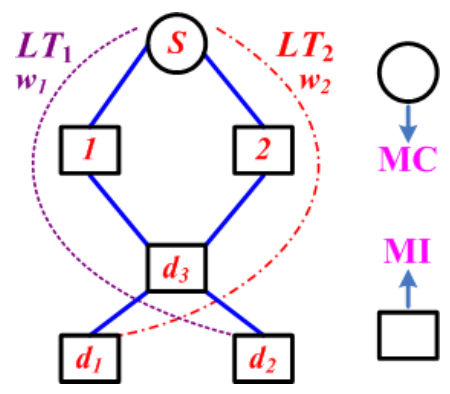

Fig. 1. An example sparse splitting WDM network

Although many light-tree computation heuristics have been proposed in recent works 391011, no literature has addressed the cost bound of multicast lighttrees in sparse splitting WDM networks, let alone the approximation of the light-trees built by heuristics towards the optimal solution. Since the wavelength channel cost is a very important metric for the selection of the multicast lighttrees, it is very interesting and imperative to know at least the cost bound of the light-trees, which could be referenced when designing a WDM network. In [11, a heuristic is proposed to construct multicast light-trees with QoS guarantee and the cost upper bound of the light-trees is given. However, in [1] it is supposed that all the network nodes are equipped with costly light splitters, while it is not realistic in large WDM mesh networks due to the high price and complex architecture of light splitters. Literature [12] also gives a cost upper bound of $\frac{N^{2}}{4}$ for the multicast light-trees in sparse splitting WDM networks, where $N$ denotes the number of nodes in the network. However, the cost bound in 12 have the following two shortcomings. First it is derived on the hypothesis that the set of multicast light-trees computed for a multicast session still retain a tree structure in the IP layer (i.e., when all these light-trees are merged together). In fact, this hypothesis is not always correct as demonstrated in the following example. A multicast session with source $s$ and destinations $d_{1}, d_{2}$ and $d_{3}$ is required in a sparse splitting optical network shown in Fig. 1 with solid line. Since node $d_{3}$ is an MI node, two light-trees (i.e., $L T_{1}$ (dotted line) and $L T_{2}$ (dashed line)) on 
two different wavelengths may be computed. As we can see the IP layer of the merged $L T_{1}$ and $L T_{2}$ are drawn in Fig. 1 with solid line, which is the same as the network topology. Obviously, it is not a tree but it exists a cycle. Second, the bound $\frac{N^{2}}{4}$ in [12 seems too large for small size multicast sessions, e.g., a multicast session with a source and only two destinations.

For the reasons above, in this paper we give a much better bound for wavelength channel cost of multicast light-trees. It is valid for most of the multicast routing algorithms under sparse splitting constraint, even if the IP layer of the set of multicast light-trees does not retain the tree structure (e.g, the iterative multicast routing algorithms as Member-Only [3]). Costly and complex wavelength converters are supposed to be unavailable, and an equal cost of 1 unit hop-count cost is assumed over all the fiber links in the network. We prove that the total cost of a multicast session is upper bounded to (1) $K(N-K)$, when $K<\frac{N}{2}$; (2) $\frac{N^{2}-1}{4}$, when $K \geq \frac{N}{2}$ and $N$ is odd; (3) $\frac{N^{2}}{4}$ when $K \geq \frac{N}{2}$ and $N$ is even, where $K$ is the number of destinations in the multicast session and $N$ is the number of nodes in the network. Besides, the total cost is lower limited to $K$. Moreover, in WDM rings the optimal multicast light-tree has a total cost inferior to $N-\left\lceil\frac{N}{K+1}\right\rceil$.

The rest of this paper is organized as follows. System model is given and the multicast routing problem is formulated in Section 2 . Then the cost bound of multicast light-trees in WDM mesh network is discussed in Section 3 After that, the cost bound of multicast light-trees in WDM rings is investigated in Section 4. Numerical results are obtained through simulation in Section 5. Finally, we conclude the paper in Section 6 .

\section{Multicast Routing with Sparse Splitting}

\subsection{Multicast Routing Problem}

Multicast routing involves a source and a set of destinations. In sparse splitting WDM networks, a set of light-trees is employed to distribute messages from the source to all the group members simultaneously. The objective of studying multicast routing in WDM networks is to minimize the wavelengths channel cost while fulfilling a multicast session. The computation of light-trees for a multicast session generally has the following principles.

1. Due to sparse splitting and absence of wavelength conversion, the degree of an MI node in the light-tree cannot exceed two. In consequence not all destinations could be included in the same light-tree. Thus, several light-trees on different wavelengths may be required for one multicast session.

2. Among the light-trees built for a multicast session, one destination may be spanned (used to forward the incoming light beam to other destination nodes) by several light-trees, but it should be served (used to receive messages from the source) by only one light-tree. (e.g., $d_{3}$ in Fig. 1 is spanned by both $L T_{1}$ and $L T_{2}$ to forward the incoming light beam to $d_{2}$ and $d_{1}$ respectively. But it must tap the light beam only once for recovering multicast messages either in $L T_{1}$ or in $L T_{2}$ ). 
3. Since the number of wavelengths supported per fiber link is limited, the maximum number of wavelengths required and the traffic congestion in a fiber link should be taken into account during the selection of multicast light-trees. Thus, if a set of destinations $D$ have been spanned by a lighttree $L T_{1}, D \subseteq L T_{1}$, it is entirely useless to construct another light-tree $L T_{2}$ to serve and only serve the destinations in subset $D_{i}$, with $D_{i} \subseteq D$. The reason is that destinations in $D_{i}$ could be served directly in $L T_{1}$.

\subsection{System Model}

A sparse splitting WDM network can be modeled by an undirected graph $G(V, E, c) . V$ represents the vertex-set of $G,|V|=N$. Each node $v \in V$ is either an MI or an MC node.

$$
V=\{v \mid v=M I \text { or } v=M C\}
$$

$E$ represents the edge-set of $G$, which corresponds to the fiber links between the nodes in the network. Each edge $e \in E$ is associated with a cost function $c(e)$. Function $c$ is additive over the links of a lightpath $L P(u, v)$ between two nodes $u$ and $v$, i.e.,

$$
c(L P(u, v))=\sum_{e \in L P(u, v)} c(e)
$$

We consider a multicast session $m s(s, D)$, which requests for setting up a light distribution structure (i.e., light-tree) under optical constraint (i.e., wavelength continuity, distinct wavelength, sparse splitting and lack of wavelength conversion constraints) from the source $s$ to a group of destinations $D$. Let $K$ be the number of destinations, $K=|D|$. Without loss of generality, it is assumed that $k$ light-trees $L T_{i}\left(s, D_{i}\right)$ are required to serve all the destinations involved in multicast session $m s(s, D)$, where $i \in[1, k]$. It holds true that

$$
1 \leq k \leq K \leq N-1
$$

Although the $i^{t h}$ light-tree $L T_{i}\left(s, D_{i}\right)$ may span some destinations already spanned in the previous light-trees, $D_{i}$ is used to denote exclusively the set of newly served destinations in $L T_{i}\left(s, D_{i}\right)$. Since all the destinations in $D$ are served by $k$ light-trees, we obtain

$$
D=\bigcup_{i=1}^{k} D_{i}
$$

These $k$ sets of destinations $D_{i}$ are disjoint, i.e.,

$$
\forall i, j \in[1, k] \text { and } i \neq j, D_{i} \cap D_{j}=\varnothing
$$

Let a positive integer $K_{i}=\left|D_{i}\right|$ denote the size of the subset $D_{i}$, then we have

$$
\sum_{i=1}^{k} K_{i}=|D|=K
$$


The total cost of multicast session $m s(s, D)$ is defined as the wavelength channel cost of the light-trees built to serve all the destinations in set $D$. It can be calculated by

$$
\begin{aligned}
c(m s(s, D)) & =\sum_{i=1}^{k} c\left[L T_{i}\left(s, D_{i}\right)\right] \\
& =\sum_{i=1}^{k} \sum_{e \in L T_{i}\left(s, D_{i}\right)} c(e)
\end{aligned}
$$

In this paper, we only investigate the cost bounds in link equally-weighted WDM networks. It is assumed that all links have the same cost function

$$
c(e)=1 \text { unit hop-count-cost }
$$

Thus,

$$
c(m s(s, D))=\sum_{i=1}^{k} \sum_{e \in L T_{i}\left(s, D_{i}\right)} 1
$$

\section{Cost Bounds of Multicast Light-Trees in WDM Mesh Networks}

In this section, we will study the cost bounds of light-trees in WDM networks with two different light splitting configurations: full light splitting and sparse splitting. Let $S R=N_{M C} / N$ be the ratio of MC nodes in the network. For the full light splitting case $S R=1$, and for the sparse splitting case $0 \leq S R<1$. Next, we first invest the cost bounds in the full splitting WDM networks.

\subsection{Full Light Splitting WDM Networks}

In the case that all network nodes are equipped with light splitters, each node could act as a branching node in a light-tree. Hence, one light-tree is sufficient to span all the multicast members. It is a Steiner-problem which tries to find a minimum partial spanning tree covering the source and all the multicast members. In a light-tree, there are at most $N$ nodes when all the networks nodes are spanned $(\{n \mid n \in L T\}=V)$, and at least $K+1$ nodes if and only if the light-tree just contains the source and the multicast members $(\{n \mid n \in L T\}=\{s\} \cup D)$. So, the cost of the multicast light-tree is bounded to

$$
K \leq c(m s(s, D)) \leq N-1
$$

To minimize the total cost in full light splitting case, the Minimum Path heuristic [6] and the Distance Network heuristic [8] can be good choices, since they are guaranteed to get a light-tree with a total wavelength channel cost no more than $2\left(1-\frac{1}{K+1}\right)[7]$ times that of the optimal Steiner tree. i.e.,

$$
c(m s(s, D)) \leq 2\left(1-\frac{1}{K+1}\right) \times C_{O p t}
$$

where $C_{O p t}$ denotes the wavelength channel cost of the Steiner tree. 


\section{$3.2 \quad$ Sparse Splitting WDM Networks}

In this subsection, the cost bounds in sparse splitting WDM networks are studied. In the case of sparse splitting, only a subset of nodes can act as branching nodes in a light-trees. One light-tree may not be sufficient to accommodate all the group members simultaneously. Generally, several light-trees should be employed.

Lemma 1. Given $i, j \in[1, k]$ and $i \neq j$, at least $\exists$ a destination $d \in D_{i}$ such that $d \notin L T_{j}\left(s, D_{j}\right)$.

Proof. The aim of constructing the $i^{t h}$ light-tree $L T_{i}\left(s, D_{i}\right)$ is to serve the destinations in the subset $D_{i}$, and the $j^{\text {th }}$ light-tree $L T_{j}\left(s, D_{j}\right)$ is used for serving the destinations in subset $D_{j}$. Let us suppose proof by contradiction that all the destinations in $D_{i}$ are also included in $L T_{j}\left(s, D_{j}\right)$, i.e., $D_{i} \subseteq L T_{i}\left(s, D_{i}\right)$ and $D_{i} \subseteq L T_{j}\left(s, D_{j}\right)$. Then, all the destinations in set $D_{i} \cup D_{j}$ can be served by only one light-tree, i.e., $L T_{j}\left(s, D_{j}\right)$. According to the third principle of multicast light-tree computation, it is entirely useless to employ an additional light-tree to re-serve the destinations in $D_{i}$. As a result, $L T_{i}\left(s, D_{i}\right)$ can be eliminated and only $k-1$ light-trees are required for multicast session $m s(s, D)$, which contradicts with the assumption. Hence, Lemma 1 is proved.

Lemma 2. $\forall j \in[1, k]$, the cost of the $j^{\text {th }}$ light-tree holds

$$
K_{j}=\left|D_{j}\right| \leq c\left(L_{j}\left(s, D_{j}\right)\right) \leq N-k
$$

Proof. According to equation (5), all the $k$ subsets of destinations $D_{i}, i \in[1, k]$, are disjoint. Based on Lemma 1 at leat $k-1$ destinations are not spanned in a light-tree. The number of nodes in a light-tree is consequently no more than $N-(k-1)$. Furthermore, if no other nodes are included in the $j^{\text {th }}$ light-tree except the source $s$ and the destinations in $D_{j}$ (i.e. $\left\{n \mid n \in L T_{j}\left(s, D_{j}\right)\right\}=\{s\} \cup D_{j}$ ), then the number of nodes in the $j^{t h}$ light-tree is minimal and equals $K_{j}+1$. Hence, the cost bounds of a light-tree can be obtained as

$$
K_{j} \leq c\left(L T_{j}\left(s, D_{j}\right)\right) \leq N-k
$$

Theorem 1. In sparse splitting WDM networks, the total cost of the light-trees built for the multicast session $m s(s, D)$ satisfies

$$
K \leq c(m s(s, D)) \leq \begin{cases}K(N-K), & K<\frac{N}{2} \\ \frac{N^{2}}{4}, & K \geq \frac{N}{2} \text { and } N \text { is even } \\ \frac{N^{2}-1}{4}, & K \geq \frac{N}{2} \text { and } N \text { is odd }\end{cases}
$$

Proof. According to Lemma 2 and equation (7), the total cost of the light-trees built for a multicast session $m s(s, D)$ holds

$$
\begin{aligned}
c(m s(s, D)) & \leq \sum_{i=1}^{k}(N-k) \\
& \leq k(N-k) \\
& \leq-\left(k-\frac{N}{2}\right)^{2}+\frac{N^{2}}{4}
\end{aligned}
$$




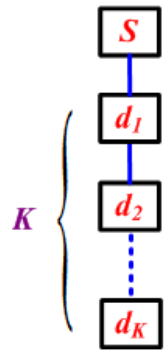

(a)

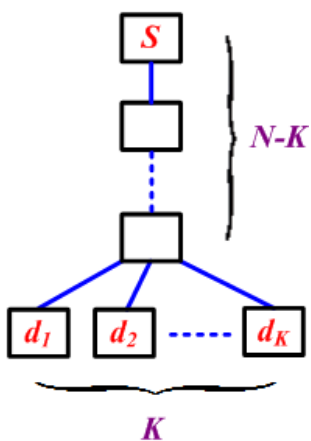

(b)

Fig. 2. (a) The best case; (b)The worst case

Regarding $k$ is an integer and $1 \leq k \leq K$, we obtain

$$
c(m s(s, D)) \leq \begin{cases}K(N-K), & K<\frac{N}{2} \\ \frac{N^{2}}{4}, & K \geq \frac{N}{2} \text { and } N \text { is even } \\ \frac{N^{2}-1}{4}, & K \geq \frac{N}{2} \text { and } N \text { is odd }\end{cases}
$$

Moreover, according to Lemma 2, it is also true that

$$
c(m s(s, D)) \geq \sum_{i=1}^{k} K_{i}=K
$$

In fact the cost bounds given in Theorem 1 are tight. In the following we give two examples to show their accuracy. It is not difficult to imagine that the case with the minimal cost appears when all and only all the destinations are involved in the light-tree computed for multicast session $m s(s, D)$, as shown in Fig. 2(a). That is to say $\{n \mid n \in L T\}=\{s\} \cup D$. It is obvious that the lower bound $K$ is tight.

And the worst case may happen when the network topology is like that in Fig. 2(b), where $K$ lightpaths on different wavelengths are needed to serve $K$ destinations to the source. Here, it is observed that the cost of the optimal light-trees equals $K(N-K)$. If the multicast session group size is equal to $|D|=K=\frac{N}{2}$ ( $K$ is even), an exact wavelength channel cost of $\frac{N^{2}}{4}$ should be consumed to establish the multicast session $m s(s, D)$. This example verifies that the exact accuracy of the upper bound given in Theorem 1 .

\section{Cost Bound of Multicast Light-Trees in WDM Rings}

\subsection{Multicast Light-Tree in WDM Rings}

In WDM rings, all the nodes are mandatorily equipped with $\mathrm{TaC}$ 4] capability, one light-tree is able to span all the multicast members. The multicast lighttree in a WDM ring consists of either a single lightpath or two edge disjoint 
lightpaths originated from the same source. In a $N$ nodes WDM ring, the cost of the multicast light-tree for multicast session $m s(s, D)$ complies

$$
K \leq c(m s(s, D)) \leq N-1
$$

\subsection{Optimal Multicast Light-Tree in WDM Rings}

Different from WDM mesh networks, minimizing the cost of multicast light-tree in a WDM ring is very simple. The minimum spanning tree for the multicast members is the optimal solution. Here, we use the concept gap introduced in 1314. A gap is a path between two adjacent multicast members in $\{s\} \cup D$ such that no other members are involved in this path nor is the source node. The optimal multicast light-tree can be obtained by removing the biggest gap from the ring $[13$.

Theorem 2. In a WDM ring, the cost of the optimal light-tree for multicast session $\mathrm{ms}(s, D)$ complies

$$
K \leq c(m s(s, D)) \leq N-\left\lceil\frac{N}{K+1}\right\rceil
$$

Proof. Beginning from the source node $s$, we index the destination nodes from $d_{1}$ to $d_{K}$ in the clockwise manner. Let $g_{1}$ denote the length of the gap between source $s$ and $d_{1}, g_{i}$ be the length of the $i^{t h}$ gap, i.e., the gap between $d_{i-1}$ and $d_{i}$, and $g_{K+1}$ be the gap between source $s$ and $d_{K}$ as shown in Fig. 3, In a WDM ring of $N$ nodes, we obtain

$$
\sum_{i=1}^{K+1} g_{i}=N
$$

And the cost of the optimal multicast light-tree for multicast session $m s(s, D)$ can be determined by

$$
c(m s(s, D))=N-\max _{1 \leq i \leq K+1} g_{i}
$$

In order to obtain the cost bound of the light-tree, we have to determine the value range of $\max _{1 \leq i \leq K+1} g_{i}$. Note that all $g_{i}$ are positive integers and satisfy equation (20). We obtain the following inequality

$$
\max _{1 \leq i \leq K+1} g_{i} \geq\left\lceil\frac{N}{K+1}\right\rceil
$$

This result corresponds to the case that multicast members are evenly distributed in the WDM ring. Thus we obtain

$$
c(m s(s, D)) \leq N-\left\lceil\frac{N}{K+1}\right\rceil
$$

Besides, if all the multicast group members stick together one by one, the optimal light-tree thus only consists of the source and the destinations. Then, we can obtain the lower bound

$$
c(m s(s, D)) \geq K
$$




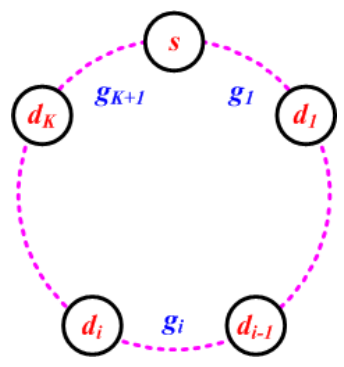

Fig. 3. The gaps in a WDM ring

\section{Simulation and Numerical Results}

As proven in Section 3.2 , the cost bound of Theorem 1 derived in sparse slitting WDM networks is valid for a serial of algorithms which respect the sparse splitting constraint plus the 3 principles mentioned in section 2. In this section, we try to obtain some numerical results to compare the proposed cost bounds and the cost of multicast light-trees computed by some classical heuristic algorithms, such as Reroute-to-Source (R2S) [3] and Member-Only (MO) 3]. Althought the cost bounds in Theorem 1 is proven to be strictly tight, the cost of multicast light-trees depends a lot on the network topologies and the distribution of the multicast session members. Hence, here we do not mean to verify the accuracy of the proposed cost bound, but just to demonstrate the difference between the cost bound and the real cost of heuristic algorithms over a simple mesh topology.

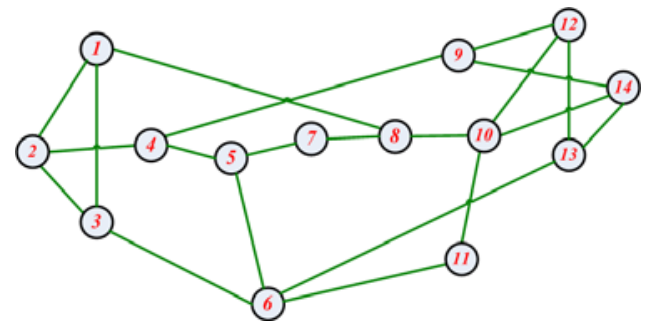

Fig. 4. The simulation topology: NSF Network

In the simulation, Reroute-to-Source and Member-Only algorithms are implemented by using $\mathrm{C}++$ with LEDA package [15] in the 14 nodes NSF network (refer to Fig. (4). The network is configured without light splitters. The source and multicast members are assumed to be distributed uniformly over the topology.

The cost bounds of the multicast light-trees computed by MO and R2S heuristics are demonstrated in Fig. [5] when the multicast group size $K+1$ varies from 2 (Unicast) to 14 (Broadcast). 5000 multicast sessions are randomly generated for 


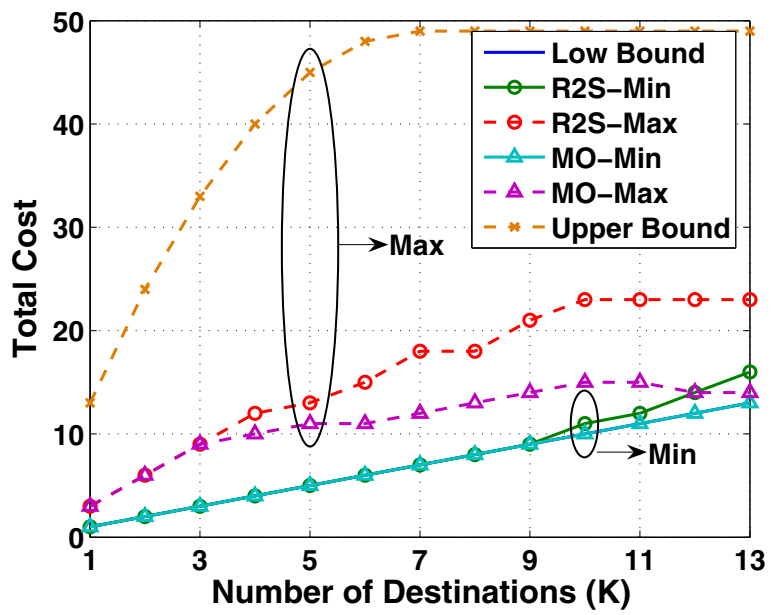

Fig. 5. The Cost Bound of multicast light-trees when the number of destinations $K$ varies in NSF Network

a given multicast group size, meanwhile Member-Only and Reroute-to-Source algorithms are employed to compute the multicast light-forest for each session. Among 5000 light-forests, the biggest cost of the light-forests (denoted by R2SMax and MO-Max) and smallest cost of the light-forests (denoted by R2S-Min and MO-Min) are figured out and plotted in Fig. 5. The low bound and the upper bound provided in Theorem 1 are compared with the simulation result. According to the figure, it is observed that the proposed low bound is covered by MO-Min since they are almost the same. The low bound is also very near to R2S-Min. But we can also find that the upper bound is much bigger than the biggest costs obtained (MO-Max and R2S-Max) by the simulation.

Table 1. Cost Bound Comparison in NSF Network

\begin{tabular}{||c||c|c|c|c|c||}
\hline \hline$|D|=K$ & LB & ILP & MO & R2S & UB \\
\hline \hline 2 & 2 & 3.2 & 3.2 & 3.6 & 24 \\
\hline 3 & 3 & 4.5 & 4.6 & 5.2 & 33 \\
\hline 4 & 4 & 5.7 & 5.7 & 6.7 & 40 \\
\hline 5 & 5 & 6.7 & 6.9 & 8.2 & 45 \\
\hline 6 & 6 & 8.2 & 8.5 & 9.1 & 48 \\
\hline 7 & 7 & 8.3 & 8.5 & 10.9 & 49 \\
\hline 8 & 8 & 8.7 & 9.3 & 11.7 & 49 \\
\hline 9 & 9 & 9.6 & 10.1 & 12.3 & 49 \\
\hline 10 & 10 & 10.8 & 11.1 & 15 & 49 \\
\hline 11 & 11 & 11.3 & 11.7 & 17.3 & 48 \\
\hline 12 & 12 & 12 & 12 & 17.3 & 49 \\
\hline 13 & 13 & 13 & 13.1 & 18.9 & 49 \\
\hline \hline
\end{tabular}


The cost bound and the average cost of 20 multicast sessions is shown in Table1, for each $K \in[2,13]$. In the table, LB denotes the lower bound, UB denotes the upper bound, and ILP denotes the average cost of the cost optimal multicast light-trees for 20 multicast sessions. The optimal multicast light-trees are computed by the integer linear programming (ILP) method by using Cplex [16]. We can see as the group size grows, the cost of any solutions (heuristic solutions and optimal ILP solutions) increases also. While the cost bound augments at the beginning, but keeps constant after $K \geq=\frac{N}{2}=7$.

Does the numerical results obtained above mean that the proposed upper bound is too big? No! This is because the simulation results depend on the simulation topology. The proposed upper bound is valid for all the algorithms which complies the three rules mentioned in section 2. As discussed in subsection 3.2 given the network topology in Figs. 2(a) and (b), both the low bound and the upper bound are always tight.

\section{Conclusion}

Multicast routing in all-optical WDM mesh networks is an important but challenging problem. It is NP-complete to minimize the wavelength channel cost consumed per multicast session under the sparse splitting constraint. Although many papers have focused on the algorithms of multicast light-trees computation, no one addresses the cost bound of light-trees nor the approximation ratios of heuristic algorithms. Hence, in this paper we investigate the bound of wavelength channel cost consumed by a multicast session. An equal cost of 1 unit hop-count cost is assumed over all the fiber links in the network. We find that it is tightly lower limited to the number of destinations $K$, and strictly upper bounded to (1) $K(N-K)$ when $K<\frac{N}{2}$; (2) $\frac{N^{2}-1}{4}$ or $\frac{N^{2}}{4}$ respectively when $K \geq \frac{N}{2}$ and $N$ is odd or even, where $K$ is the number of destinations in the multicast session and $N$ is the number of nodes in the network. Source-oriented multicast light-trees computation heuristic algorithms like Reroute-to-Source [3] and Member-Only 3 follow this cost bound, as they respect the three principles for light-trees computation mentioned in Section 2, In a particular situation, where the network topology is a WDM ring, the optimal multicast light-tree can be determined by removing the biggest gap from the ring. We found its cost is inferior to $N-\left\lceil\frac{N}{K+1}\right\rceil$. In the future, the approximation ratios of some classical heuristic algorithms of all-optical multicast routing will be investigated.

\section{References}

1. Sahasrabuddhe, L.H., Mukherjee, B.: Light-trees: optical multicasting for improved performance in wavelength-routed networks. IEEE Communications Magazine 37(2), 67-73 (1999)

2. Malli, R., Zhang, X., Qiao, C.: Benefit of multicasting in all-optical networks. In: SPIE Proceeding on All-Optical Networking, vol. 2531, pp. 209-220 (1998) 
3. Zhang, X., Wei, J., Qiao, C.: Constrained multicast routing in WDM networks with sparse splitting. IEEE/OSA Journal of Lightware Technology 18(12), 1917-1927 (2000)

4. Ali, M., Deogun, J.S.: Cost-effective Implementation of Multicasting in Wavelength-Routed Networks. IEEE/OSA Journal of Lightwave Technology 18(12), 1628-1638 (2000)

5. Mukherjee, B.: WDM optical communication networks: progress and challenges. IEEE Journal on Selected Areas in Communications 18(10), 1810-1824 (2000)

6. Takahashi, H., Matsuyama, A.: An approximate solution for the Steiner problem in graphs. Math. Japonica 24(6), 573-577 (1980)

7. Winter, P.: Steiner Problem in Networks: A Survey. Networks 17, 129-167 (1987)

8. Kou, L., Markowsky, G., Berman, L.: A fast algorithm for Steiner trees. Acta Informatica 15, 141-145 (1981)

9. Zhou, F., Molnár, M., Cousin, B.: Avoidance of Multicast Incapable Branching Nodes in WDM Netwoks. Photonic Network Communications 18(3), 378-392 (2009)

10. Zhou, F., Molnár, M., Cousin, B.: Is light-tree structure optimal for multicast routing in sparse light splitting WDM networks. In: The 18th International Conference on Computer Communications and Networks, San Francisco, USA, August 2, pp. $1-7(2009)$

11. Jia, X., Du, D., Hu, X., Lee, M., Gu, J.: Optimization of wavelength assignment for QoS multicast in WDM networks. IEEE Transaction on Communications 49(2), 341-350 (2001)

12. Lin, H., Wang, S.: Splitter placement in all-optical WDM networks. In: Proceeding of IEEE GLOBECOM, pp. 306-310 (2005)

13. Scheutzow, M., Seeling, P., Maier, M., Reisslein, M.: Multicasting in a WDMupgraded resilient packet ring. Journal of Optical Networking 6(5), 415-421 (2006)

14. Scheutzow, M., Seeling, P., Maier, M., Reisslein, M.: Shortest path routing in optical WDM ring networks under multicast traffic. IEEE Communications Letters 10(7), 564-566 (2006)

15. http://www.algorithmic-solutions.com/leda/index.htm

16. http://www.ilog.com/products/cplex/ 